\title{
Intersystem crossing as a key component of the non-adiabatic relaxation dynamics of bithiophene and terthiophene
}

Thomas Schnappinger, ${ }^{\dagger}$ Marco Marazzi, ${ }^{\dagger}$ Sebastian Mai, ${ }^{\S}$ Antonio Monari, Leticia González, ${ }^{\S}$ and Regina de Vivie-Riedle*,†

Department of Chemistry, Ludwig-Maximilians-Universität München, D-81377 München, Germany, Departamento de Química, Centro de Investigación en Síntesis Química (CISQ), Universidad de La Rioja, 26006 Logroño, Spain, Université de Lorraine and CNRS, LPCT UMR 7019, F-54000 Nancy, France, and Institute of Theoretical Chemistry, Faculty of Chemistry, University of Vienna, 1090 Vienna, Austria

E-mail: Regina.de_Vivie@cup.uni-muenchen.de

\begin{abstract}
We present a non-adiabatic dynamics study concerning the sub picosecond relaxation of excited states in dimeric and trimeric thiophene chains. The influence of the triplet states in the overall process is, for the first time, taken into account by explicitly including spin-orbit couplings and hence allowing intersystem crossing phenomena. We observe the fundamental role of the triplet state manifold in driving the full relaxation

${ }^{*}$ To whom correspondence should be addressed

†Department of Chemistry, Ludwig-Maximilians-Universität München, D-81377 München, Germany

${ }_{\ddagger}^{\ddagger}$ Departamento de Química, Centro de Investigación en Síntesis Química (CISQ), Universidad de La Rioja, 26006 Logroño, Spain

๑ Université de Lorraine and CNRS, LPCT UMR 7019, F-54000 Nancy, France

$\S$ Institute of Theoretical Chemistry, Faculty of Chemistry, University of Vienna, 1090 Vienna, Austria
\end{abstract}


process. In particular we evidence the effect of both, inter-ring rotation and ringopening, in the process, as compared to the monomer, where the ring-opening process appears as the dominant one. In addition, the evolution of the open structures allows for trans to cis isomerization in the dimer and trimer. The overall relaxation process slows down with chain elongation. The complex decay mechanism characterized by the presence of different competing channels, due to the presence of a quasi degenerate manifold, is explained allowing the rationalization of oligothiophenes photophysics.

\section{Introduction}

Due to their remarkable intrinsic properties thiophene derivatives and their oligomers are promising candidates to be employed in organic materials for technological applications. ${ }^{1,2}$ They undergo rapid intersystem crossing (ISC) ${ }^{3-5}$ leading to extremely high triplet quantum yields. ${ }^{6}$ This property deviates strongly from the common behavior found in most organic molecules where the ratio of ISC to internal conversion (IC) and fluorescence is rather small. ${ }^{7}$ Also, it is important for charge separation and transport in conductive organic materials. In combination with their efficient light harvesting and structural versatility they have attracted much attention as building blocks for photoactive polymers and molecular aggregates. Examples are solar cells, ${ }^{8-12}$ light emitting diodes, ${ }^{13,14}$ photoswitches ${ }^{15}$ and biological labels. ${ }^{16-18}$ In addition, polythiophene bridges are also used to enhance two-photon absorption cross sections. ${ }^{19-21}$ As poly(3-hexylthiophene) (P3HT) they form in combination with phenyl-C61-butyric acid methyl ester (PCBM) the famous P3HT/PCBM solar cell. ${ }^{11,22}$ A deeper understanding of the initial processes after photoexcitation and the subsequent relaxation mechanisms of oligothiophenes is essential to improve the performance of thiophenebased applications. In our previous works ${ }^{23,24}$ we were able to explain the relaxation processes for the monomer thiophene with the aid of static quantum chemical calculations and non-adiabatic molecular dynamics simulations: after photoexcitation, fast ring-opening (approx. $100 \mathrm{fs}$ ) leads to a degeneracy of singlet and triplet states. This degeneracy and the 
corresponding flat potential energy surface traps the system in entropically favored open-ring structures in the singlet as well as in the triplet states (up to $50 \mathrm{ps).}{ }^{25}$

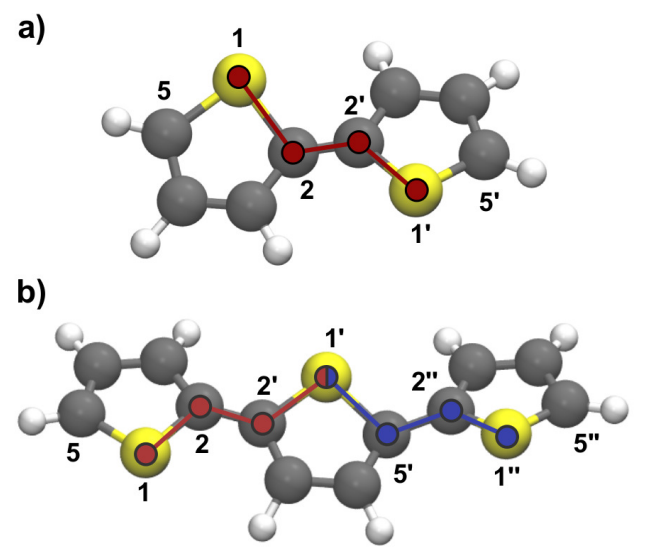

Figure 1: Structure of 2,2'-bithiophene (TP2) in the trans conformation a) and of $2,2^{\prime}: 5$ ',2"-terthiophene (TP3) in the eclipsed-anti-anti (eAA) conformation b). All relevant atoms are labeled, as well as the inter-ring dihedral angles: $\Phi_{S C C S}$ (red) in TP2, $\Phi_{S C C S}^{1}$ (red) and $\Phi_{S C C S}^{2}$ (blue) in TP3, respectively. The nomenclature is explained in more detail in the Supporting Information $\left(\mathrm{SI}^{\dagger}\right)$.

The structures of the most stable conformer are shown in Figure 1 for the dimer TP2 (2,2'-bithiophene) and the trimer TP3 (2,2':5',2"'-terthiophene). For both TP2 and TP3 experimental results ${ }^{6,26-28}$ indicate that ultrafast ISC takes place from the first excited singlet state. Moreover experimental and theoretical studies suggested two ISC channels for both systems with timescale ranging from 1 ps up to 140 ps. ${ }^{26,27}$ Based on static quantum chemical calculations ${ }^{23,29 ?-32}$ and dynamics simulations (excluding triplet states) ${ }^{33,34}$ the relaxation processes after photo excitation for TP2 and TP3 are mainly ascribed to the torsional motion around the quite flexible inter-ring bonds, ${ }^{35}$ whereas ring-opening via CS-bond cleavage should only play a minor role in the initial relaxation processes of both systems. However, for the previous dynamics simulations ${ }^{33,34}$ only short simulation times (300-500 fs) were studied and important ISC processes were excluded.

This work presents the first non-adiabatic dynamical study of the dimer TP2 and the trimer TP3 at picosecond timescale explicitly including spin-orbit couplings and triplet states. For this study we have used the surface hopping including arbitrary couplings (SHARC) soft- 
ware package, ${ }^{36-39}$ which has already been successfully applied to an important number of molecular systems. ${ }^{24,40-44}$

\section{Computational methods}

\section{Ab initio level of theory}

The electronic states of TP2 and TP3 were computed using the density functional theory (DFT) and linear response time dependent DFT (LR-TDDFT). All LR-TDDFT calculations have been performed within the so-called Tamm-Dancoff approximation (TDA). ${ }^{45}$ The DFT and TDA calculations were carried out with the ADF program package (version 2017), ${ }^{46,47}$ using the B3LYP ${ }^{48-50}$ exchange-correlation functional with the D3 dispersion correction by Grimme and coworkers. ${ }^{51}$ Minima (Min), transition states (TS), conical intersections (CoIn) and singlet-triplet minimum-energy crossings (STC) were optimized with the ORCA code (version 3.0.3) ${ }^{52}$ as an external optimizer. All optimized geometries are given in the Supporting Information $\left(\mathrm{SI}^{\dagger}\right)$. The DFT and TDA calculations were carried out with the the double zeta polarized basis set (DZP) ${ }^{53}$ and a Becke ${ }^{54,55}$ and Zlmfit ${ }^{56}$ grid of good quality. For benchmarking purposes, some calculations were performed with the triple zeta polarized basis set $(\mathrm{TZP})^{53}$ in combination with the same grid quality. In all optimization calculations symmetry was not used. Scalar relativistic effects were included with the zeroth-order regular approximation (ZORA), ${ }^{57-61}$ and the spin-orbit couplings (SOC) were calculated using a perturbative method, as developed and implemented by Wang and Ziegler. ${ }^{62}$

\section{Dynamics}

The molecular dynamics of TP2 and TP3 were simulated using the SHARC ab initio dynamics package version 2.0. ${ }^{36-39}$ The necessary energies, gradients, non-adiabatic couplings and spin-orbit couplings were calculated on-the-fly at the TDA/B3LYP level of theory. The initial conditions for the dynamics simulations were generated based on a Wigner distri- 
bution computed from harmonic vibrational frequencies in the optimized ground state equilibrium geometry. The underlying frequency calculations were performed at B3LYP/DZP level of theory. For TP2 only the trans conformer was studied, since it has been shown to be the ground state global minimum. ${ }^{23,63}$ Time-resolved spectroscopic studies on the dimer ${ }^{26,27}$ used an excitation energy of $4.02 \mathrm{eV}$ to reach the rising edge of the first absorption peak. Corresponding to these experiments we have centered the spectral window (3.0-4.0 eV) at the rising edge of the first absorption peak (see the simulated absorption spectrum in the $\left.\mathrm{SI}^{\dagger}\right) .84$ starting geometries and velocities were stochastically chosen.

For TP3, among the total number of ten ground state minima, ${ }^{31,64 ?-66}$ two nearly isoenergetic minima exist. ${ }^{23}$ The eclipsed-anti-anti (eAA) conformer is the global minimum and the gauche-anti-anti $(g A A)$ conformer is only $0.02 \mathrm{eV}(\Delta \mathrm{G})^{23}$ above. Since these conformers can easily interconvert, as will be shown below, we restrict our initial condition generation to the $e A A$ conformer. For the dynamics simulations of TP3, 36 starting geometries and velocities in the energy window 3.0-4.0 eV were stochastically chosen from a Wigner distribution (see the simulated absorption spectrum in the $\mathrm{SI}^{\dagger}$ ). For both systems, in accord with the chosen excitation window, all trajectories started in the $\mathrm{S}_{1}$ state.

The SHARC ab initio surface-hopping algorithm uses a fully diagonal, spin-mixed electronic basis, resulting from the diagonalization of the Hamiltonian containing spin-orbit couplings. ${ }^{37,67}$ The integration of the nuclear motion is done with the Velocity-Verlet algorithm with a maximal time of 1 ps using a time step of 0.5 fs. In each time step, gradients were computed for all states which are closer than $0.2 \mathrm{eV}$ to the active state; this is required in order to construct the gradient of the spin-mixed active state. The coefficients of the electronic wavefunction are propagated on interpolated intermediates with a time step of $0.02 \mathrm{fs}$ applying a local diabatization technique ${ }^{68}$ in combination with the WFoverlaps code ${ }^{69}$ to compute the wave function overlaps. Decoherence correction was taken into account using the energy-based method of Granucci and Persico with the parameter $\alpha=0.1$ a.u. ${ }^{70}$ For the statistical analysis of the results stemming from the non-adiabatic dynamics, the 
number of aborted trajectories has to be considered. Some trajectories experienced convergence problems and therefore terminated before reaching 1 ps. Beside the convergence, also problems arising from the DFT/TDA methodology (e.g. the multi-configurational character of the ground state) have to be taken into account since they may lead to unphysical behavior of the energy and the gradients. In order to find a good compromise between the simulation time considered and the number of trajectories inspected, we restrict our analysis to trajectories with at least 400 fs of simulation time, a maximum change in the total energy of $0.2 \mathrm{eV}$, a maximum change in the total energy per step of $0.2 \mathrm{eV}$ and a maximal inter-state hop energy of $1.0 \mathrm{eV}$.

\section{Results and discussion}

\section{Validation of the level of theory}

First, it was checked whether the static B3LYP/DZP and B3LYP/TZP results for both systems are in qualitative agreement with previous CASPT2 results ${ }^{23}$ and the experimental data. ${ }^{6,32,71-73}$ Therefore, the excitation energies of the optimized ground-state minima (trans for TP2 and $e A A$ for TP3) were benchmarked (see Table 1).

The calculated $\mathrm{S}_{1}$ and $\mathrm{S}_{2}$ excitation energies for TP2 are in between the CASPT2 and the experimental results. For the first four triplet states the B3LYP energies are in good agreement with the CASPT2 values. The energetic order and the electronic character of all tested states are the same as in previous studies. ${ }^{23,29,30,32}$ Moreover, the B3LYP functional reproduces the CASPT2 excitation energies for the first six states of TP3 quite well. The character of the states is also the same as in previous studies, ${ }^{23,31,32}$ except for a slight difference in the state ordering. Using TDA the third triplet state $T_{3}$ lies above the $S_{1}$ independently of the chosen basis set. For both systems the TZP basis set gives better agreement with experiment than the smaller basis set DZP. In order to save time for the dynamics simulations we decided to use the small DZP basis set, as it is sufficient to well 
Table 1: Calculated vertical singlet and triplet excitation energies (eV) for the low-lying excited states at the optimized ground-state minimum of TP2 and TP3 compared to experimental and theoretical data. Oscillator strength values are given in parentheses. Energies were calculated using B3LYP/DZP and B3LYP/TZP.

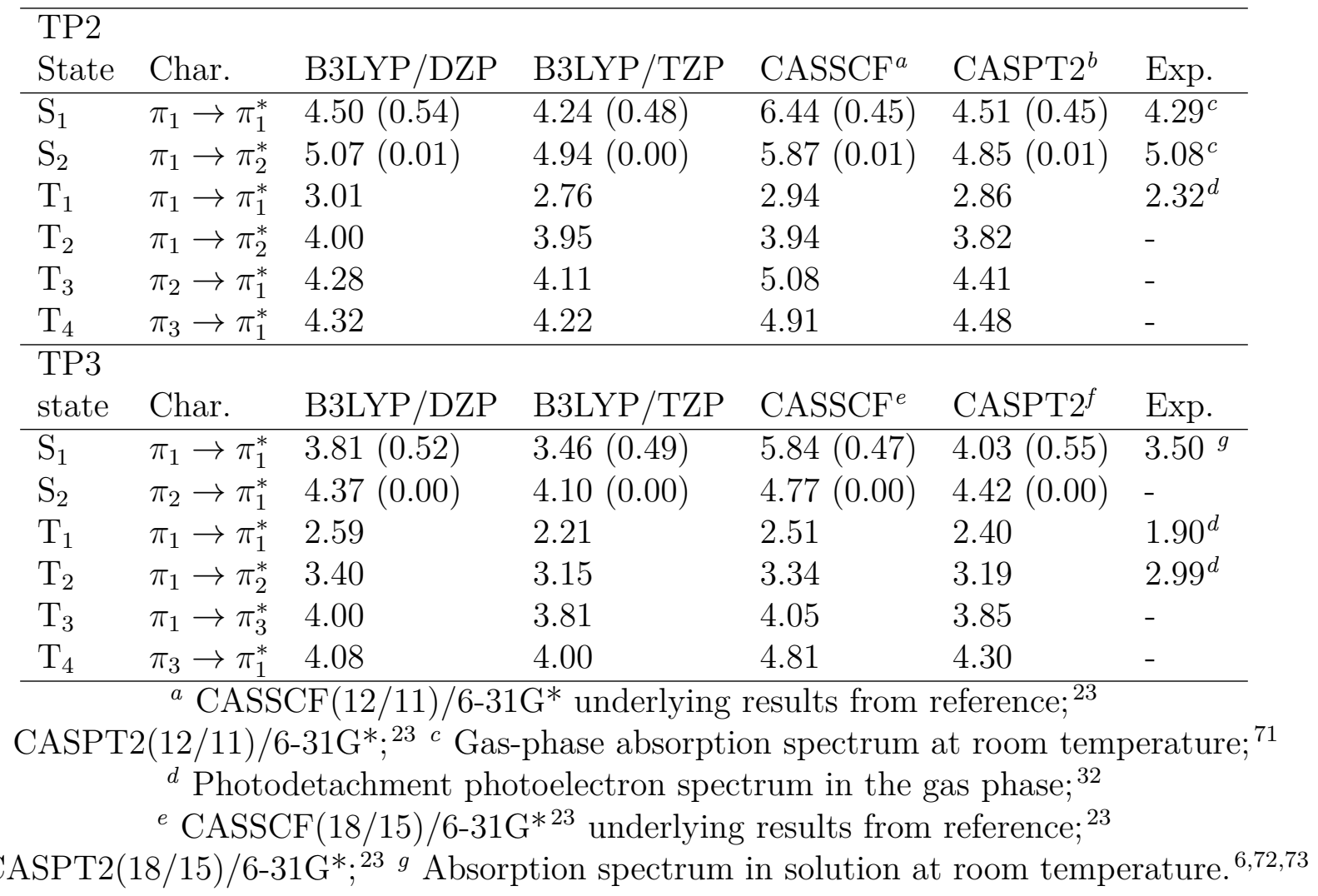

describe the low-lying excited excited states of TP2 and TP3 in the Franck-Condon region. In contrast to the CASPT2 methodology the excitation energies at the CASSCF level as well as the state ordering is wrong for TP2 and TP3. Hence, a meaningful description of TP2 and TP3 is not possible using CASSCF.

Based on static quantum chemical calculations ${ }^{23,29-32}$ two possible deactivation pathways for TP2 and also TP3 were discussed: the first pathway is described by the rotation around the inter-ring bonds. This rotation leads to a near-degeneracy region of triplet states and the $\mathrm{S}_{1}$ state. In combination with the moderate spin-orbit couplings this degeneracy should allow ISC; subsequent internal conversion steps lead the system in the $\mathrm{T}_{1}$ state. The relaxation back to the ground state can take place through either phosphorescence or non-radiative processes like cleavage of a C-S bond. The second pathway is similar to the relaxation mech- 
anism in the thiophene monomer: ${ }^{24}$ the C-S bond cleavage leads to open-ring structures where the $\mathrm{S}_{0}, \mathrm{~T}_{1}, \mathrm{~S}_{1}$ and $\mathrm{T}_{2}$ states are nearly degenerate and the $\mathrm{SOC}$ increases significantly. Via these open-ring structures, closed-ring conformers can be formed in the ground state. In principle for TP2 as well as for TP3 many open-ring structures are possible. As shown in our previous work ${ }^{23}$ structures with only one broken C-S bond are energetically favored. In TP2 the four C-S bonds can be distinguished into two groups: the inner bonds (1,2 and 1',2', see Fig. 1) and the outer bonds (1,5 and 1',5'). The cleavage of the inner bonds is energetically favored. The C-S bonds of TP3 can be distinguished analogously in three groups: for both terminal rings the C-S bonds can be divided into inner and outer ones. The C-S bonds of the central thiophene ring form the third group, the central bonds. The cleavage of one of the inner or central bonds leads again, compared to outer bonds, to more stable structures. In the following the discussion is restricted to open-ring structures with a single broken inner or central C-S bond.

All critical points of TP2 and TP3 along both relaxation pathways were optimized using the small basis set DZP. The excitation energies of the six low-lying excited states were determined and compared with previous CASPT2 calculations. ${ }^{23}$ These results are collected in the $\mathrm{SI}^{\dagger}$. For all closed-ring structures the B3LYP/DZP results are in sufficient agreement with the CASPT2 values with slightly higher deviations for open-ring structures. We are aware of the inherent problems of the TD-DFT methodology to describe multi-configurational character in the ground-state and conical intersection between $\mathrm{S}_{1} / \mathrm{S}_{0}$. However, in previous studies on oligothiophenes ${ }^{23,32-34}$ the relaxation pathway can be split into two parts. In the first part describing the path from the Franck-Condon region to the open-ring structures the triplet states lie energetically close to the $\mathrm{S}_{1}$ state and the spin-orbit coupling is large. We are very confident that this first part of the relaxtion is fully described by TD-DFT. The second part is the dynamic of the open-ring structures and the relaxation back to the ground-state. Here the conical intersection between $\mathrm{S}_{1} / \mathrm{S}_{0}$ is very important but not described well using TD-DFT. This may be biasing the simulation towards the triplet state population. On the 
other hand, the relaxation into triplet states is also present in thiophene where we could perform the dynamics simulation at the CASSCF level. ${ }^{24}$ Nevertheless, the overall shape of the potential energy surfaces along the pathways is qualitatively well reproduced using the B3LYP/DZP methodology. Therefore, we are convinced that the chosen methodology is adequate to describe the dynamics of TP2 and TP3.

\section{Excited state dynamics of 2,2'-bithiophene (TP2)}

Using our selection criteria, 53 trajectories out of 84 trajectories were taken into account to analyze the excited state dynamics of TP2. These 53 trajectories were first analyzed according to the final populated state and the final molecular geometry.A geometry is characterized as open-ring structure if one of the C-S bonds is larger than $2.4 \AA$. The results are summarized in Table 2.

Table 2: Distribution (\%) of the TP2 B3LYP/DZP trajectories according to the final populated state as well as the final geometry. All percentages are given with respect to the total number of analyzed trajectories. The shown numbers are statistically not fully converged. The maximum statistical error is $13 \%{ }^{74}$

\begin{tabular}{l|lll|l}
\hline final state & \multicolumn{3}{|c|}{ final geometry } & $\Sigma$ \\
& open & cis & trans & \\
\hline $\mathrm{S}_{0}$ & 16 & 12 & 6 & 34 \\
$\mathrm{~S}_{1}$ & 4 & 0 & 0 & 4 \\
$\mathrm{~T}_{1}$ & 24 & 4 & 10 & 38 \\
$\mathrm{~T}_{2}$ & 24 & 0 & 0 & 24 \\
\hline$\Sigma$ & 68 & 16 & 16 & 100
\end{tabular}

After excitation to the $S_{1}$ state, all analyzed trajectories show non-adiabatic relaxation within the first picosecond. The final distribution between the singlet and the triplet states is nearly $2: 3$. Within the triplet manifold the relative distribution $\mathrm{T}_{1}: \mathrm{T}_{2}$ is about $3: 2$, within the singlet moiety $\mathrm{S}_{0}: \mathrm{S}_{1}$ is 8.5:1. Most final geometries are open-ring structures (68\%), where one inner bond is broken. Closed-ring conformations (trans or cis) are only found for the $\mathrm{S}_{0}$ and the $\mathrm{T}_{1}$ state. Note that in the monomer almost no triplet closed-ring structures were found. ${ }^{24}$ Although all trajectories have been propagated from an initial trans conformation, 
a one-to-one mixture of trans and cis conformers is observed after relaxation.

The overall change in population of the ground state and the individual excited states for the first picosecond is shown in Figure 2 for the TP2 dynamics.

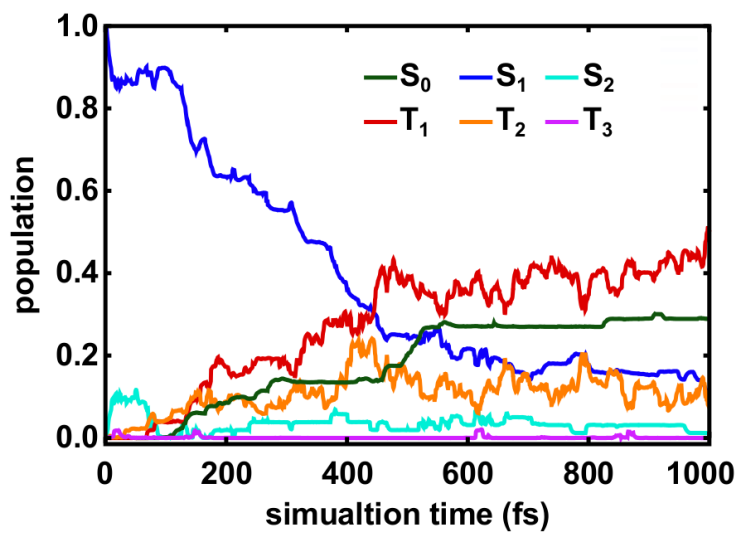

Figure 2: Time evolution of the average populations along the TP2 trajectories. The ground and the five lowest excited states are displayed.

The populations dynamics of TP2 is more complex than for the thiophene monomer ${ }^{24}$ and far from exponential behavior. It is characterized by a fast decay of the $\mathrm{S}_{1}$ state population. In the first 3 to 5 fs there is an ultrafast exchange of some population between $\mathrm{S}_{1}$ and $\mathrm{S}_{2}$ states, probably attributable to the initial conditions. Nevertheless, during the whole process the $\mathrm{S}_{2}$ state plays no role. Indeed, after this initial exchange, the $\mathrm{S}_{1}$ state population stays constant for about 100 fs. Thereafter the first two triplet states, $\mathrm{T}_{2}$ and $\mathrm{T}_{1}$, start to participate via ISC. The $S_{1}$ state is mainly depopulated via the $T_{2}$. Subsequently the $T_{1}$ state is populated via IC. This cascade $\mathrm{S}_{1} \rightarrow \mathrm{T}_{2} \rightarrow \mathrm{T}_{1}$ finally enables the population transfer back in the ground state $S_{0}$. The $T_{3}$ state is nearly not populated. The ratio between $S_{0}, S_{1}$, $\mathrm{T}_{1}$ and $\mathrm{T}_{2}$ after 1 ps simulation time is approx. $30: 10: 50: 10$. Compared to the monomer dynamics, the relaxation from $\mathrm{S}_{1}$ state is significantly slower in the first $200 \mathrm{fs}$.

Next we analyze which relaxation pathway leads to the final structures. The two most relevant internal coordinates, previously introduced, are the rotation around the inter-ring bond (i.e. the dihedral angle $\Phi_{S C C S}$ ) and the C-S bond cleavage described as the averaged C-S distance $r_{C S}$ of both inner C-S bonds. In Figure 3 the time evolution (color coded) of 
the trajectories is shown for the first 200 fs.

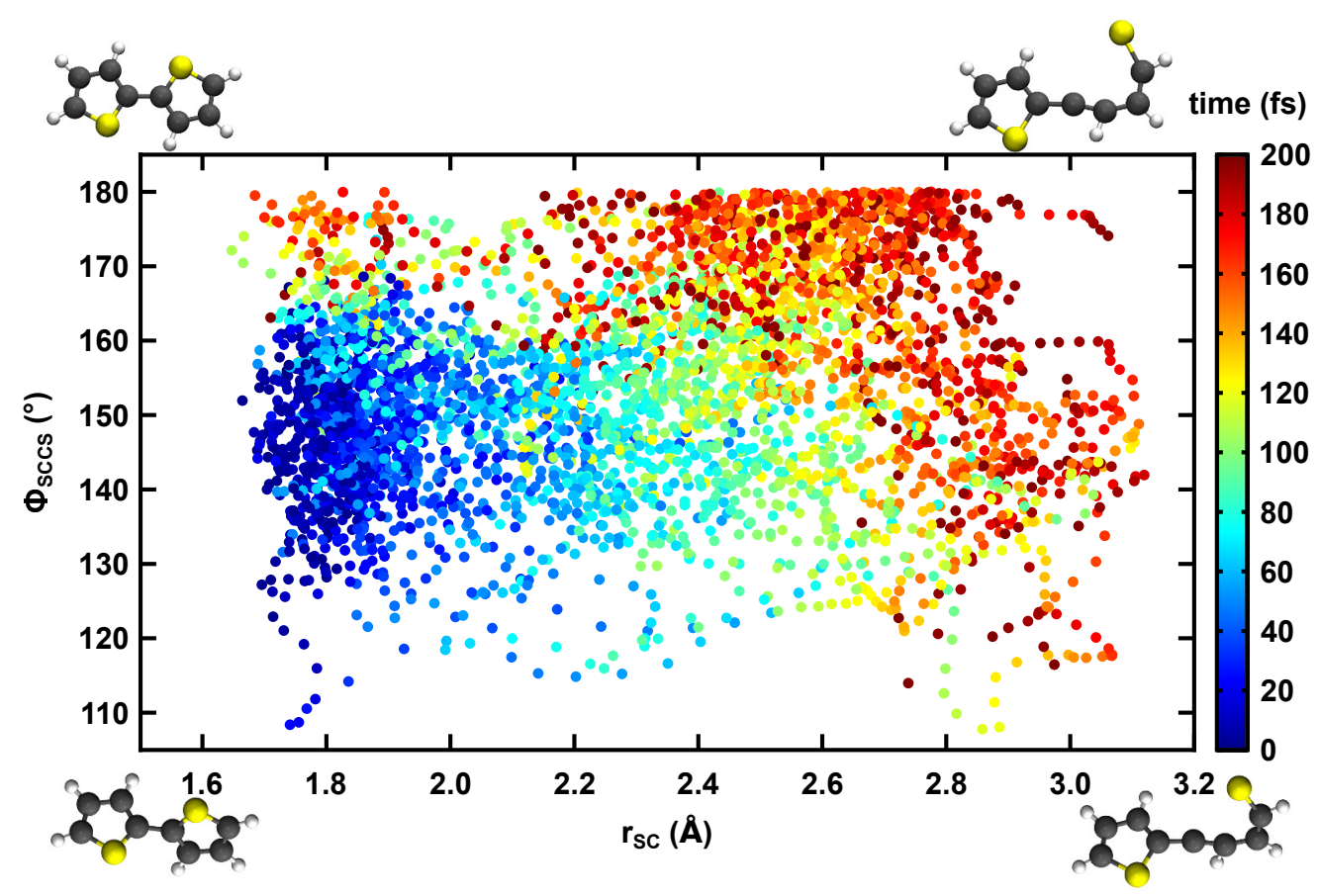

Figure 3: Analysis of the non-adiabatic dynamics of TP2 within the first 200 fs. Time evolution (see color code) of the trajectories in the subspace spanned by the averaged 'inner' C-S distance $r_{C S}$ and the dihedral angle $\Phi_{S C C S}$.

Starting from the Wigner distribution (dark blue dots) the dihedral angle $\Phi_{S C C S}$ varies between $130^{\circ}$ and $160^{\circ}\left(\mathrm{S}_{0}\right.$-minimum at $\left.143.0^{\circ}\right)$ and an averaged inner $\mathrm{C}$-S distance $r_{C S}$ is found between $1.7 \AA$ and $1.9 \AA\left(\mathrm{S}_{0}\right.$-minimum value $\left.1.75 \AA\right)$. Within the first 100 fs a small part of the trajectories leads to planarization while the majority undergoes ring-opening reaching an average bond distance of up to $2.6 \AA$. Thereafter further bond elongation is accompanied by significant planarization reaching $180^{\circ}$. The dynamics simulation shows that both reaction coordinates (ring-opening and planarization) are active on the same time scale. The energetic barrier for the ring-opening in TP2 is estimated to be $0.2 \mathrm{eV}$ (B3LYP/DZP). The maximal barrier at CCSD/6-31G* level of theory was found to be $0.4 \mathrm{eV} .{ }^{23}$ Both values are higher than for the barrier in the monomer ${ }^{24}$ found at $0.1 \mathrm{eV}$. Our dynamics simulations confirm that due to the excess kinetic energy deposited in the photon absorption process this barrier can still be overcome, but it explains the slower decay of the $\mathrm{S}_{1}$ population (see 
Fig. 2). The complete temporal evolution up to 1 ps for $\Phi_{S C C S}$ and $r_{C S}$ can be found in the $\mathrm{SI}^{\dagger}$.

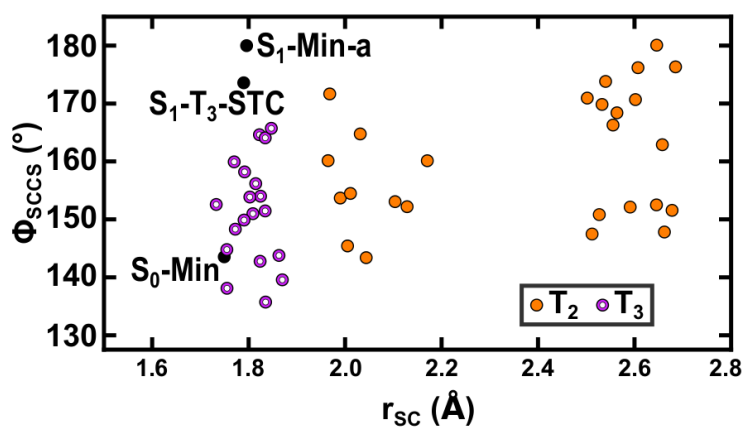

Figure 4: Locations of the geometries where the first surface hops from the $\mathrm{S}_{1}$ state to triplet states $\left(\mathrm{T}_{2}\right.$ and $\left.\mathrm{T}_{3}\right)$ occurred depending on $\mathrm{r}_{c s}$ and $\Phi_{S C C S}$ in TP2 within the first 200 fs. The hopping geometries are displayed in different colors according to the target state of the hopping event. Additionally, relevant B3LYP/DZP optimized geometries are represented in black, including minima (Min) and singlet-to-triplet crossings (STC).

The geometrical deformations observed in the first $200 \mathrm{fs}$ lead to many intersystem crossing events from the $\mathrm{S}_{1}$ state into the triplet states (see Fig. 4). Mostly transitions into the $\mathrm{T}_{2}$ and $\mathrm{T}_{3}$ state are observed, which is in agreement with previous results. ${ }^{23,34}$ In principal these hopping events can be distinguished geometrically and temporally. Hops between the $\mathrm{S}_{1}$ state and the $\mathrm{T}_{3}$ state only occur for closed-ring and non-planar geometries, hence very early. They are mainly forward and backward hops with no net populations exchange. This explains the plateau in the $S_{1}$ state population observed in Figure 2 in the range from 3 up to $100 \mathrm{fs}$. Hops to the $\mathrm{T}_{2}$ state are observed around two different $r_{C S}$ values. For the slightly elongated C-S bonds (2.0-2.2 $\AA$ ) the SOC values increase and the two states $\mathrm{T}_{2}$ and $\mathrm{S}_{1}$ cross. This initiates the decay of the $\mathrm{S}_{1}$ state after $100 \mathrm{fs}$ in Figure 2. Further elongation of $r_{C S}$ (up to 2.5-2.6 $\AA$ ) leads to a further increase in the SOC and to a near degeneracy of the $T_{2}$ and $S_{1}$ state, giving rise to a second enhancement of hops shown in Figure 4. A complete analysis of all hopping events for the whole simulation time can be found in the $\mathrm{SI}^{\dagger}$. The open-ring dynamics lead, as in the case of the thiophene monomer, ${ }^{24}$ to a dynamical equilibrium between singlet and triplet states. All states involved, $\mathrm{S}_{0}, \mathrm{~S}_{1}, \mathrm{~T}_{1}$ and $\mathrm{T}_{2}$, come 
close in energy accompanied by rather large effective spin-orbit couplings $\left(\mathrm{SI}^{\dagger}\right)$. Although the degeneracy is not as complete among all states as in case of the monomer, many back and forward hops between $S_{1}, T_{1}$ and $T_{2}$ establish the dynamical equilibrium. Due to the chosen methodology, i.e. TDDFT, hops back into the ground state are only possible via the $\mathrm{T}_{1}$ and $\mathrm{T}_{2}$ state because of a poor description of the open-ring $\mathrm{S}_{1} / \mathrm{S}_{0}$ conical intersection. This might explain why the final population in $\mathrm{S}_{0}$ is lower than in $\mathrm{T}_{1}$, while for the monomer, calculated at CASSCF level, both states were equally populated. ${ }^{24}$

The energy profiles of four illustrative trajectories are shown in Figure 5. The individual states are color coded and the populated state is marked with black diamonds.
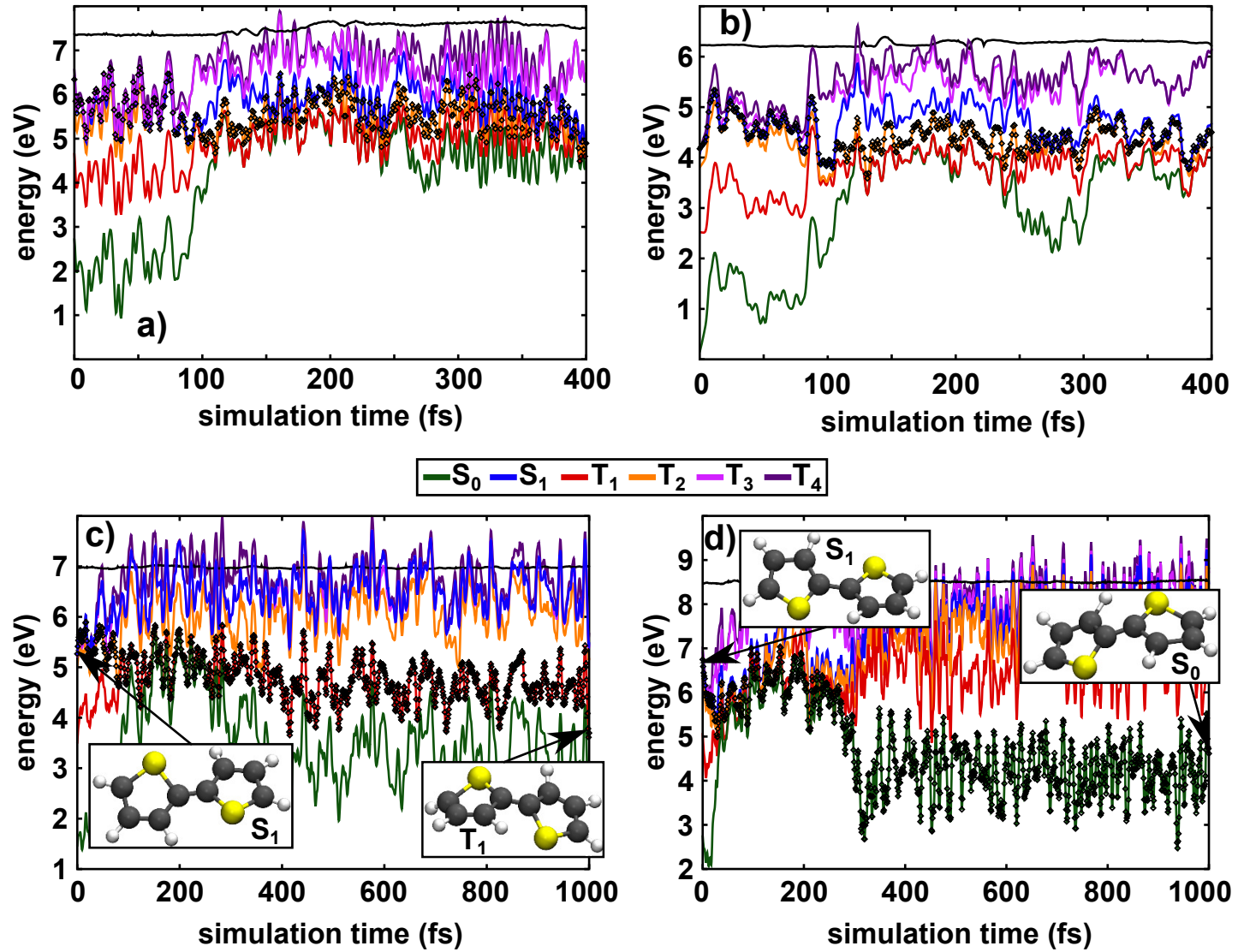

Figure 5: Energy profiles of four representative TP2 trajectories following the ring-opening mechanism. All trajectories were initiated on the $S_{1}$ potential energy surface. The time evolution of the ground and the six lowest excited states are displayed in color, whereas the running state is indicated with black diamonds. The energies are plotted relative to the ground state minimum. Initial and final structures are given for the last two trajectories. 
All four trajectories undergo ring-opening with the cleavage of one of the inner C-S bonds after the first 100 fs. In Figure 5 a) and b) we focus on the first 400 fs. In the open-ring structure, the system switches mostly between the quasi-degenerate $\mathrm{S}_{1}$ and the $\mathrm{T}_{2}$ state. In contrast to the monomer case, the ground state $S_{0}$ and the first triplet state $T_{1}$ show much less participation to the process. Two different reaction pathways leading to closed-ring structures are shown in Figure $5 \mathrm{c}$ ) and d). In example c) one C-S bond is broken after approximately 90 fs. The $T_{1}$ state is reached via $\mathrm{S}_{1}$ and $\mathrm{T}_{2} 20$ fs later. Although the $\mathrm{T}_{1}$ state and the ground state are nearly degenerate, no hops occur for the next 100 fs and the ring-closure takes place in the triplet state forming a nearly planar trans conformer. In contrast, Figure 5 d) reports an example of relaxation to a closed ring trans conformer in the $S_{0}$ state, reached from the $S_{1}$ state via the $T_{2}$ and the $T_{1}$ state. Note, that although we presented trajectories leading to trans products, the open ring structures can rotate almost freely around the inter-ring bond and hence can easily undergo isomerization into the cis conformer. This is reflected in the ratio between trans and cis products shown in Table 2. The amount of closed-ring conformers found for the $T_{1}$ state explains the experimentally observed phosphorescence ${ }^{75}$ which might occur from these stable geometries.

\section{Excited state dynamics of 2,2':5',2"'-terthiophene (TP3)}

For TP3, 34 out of 36 trajectories were taken into account for discussion of the excited state dynamics. These 34 trajectories were first analyzed according to the final populated state and the final molecular geometry. Just as for the TP2 analysis a geometry is characterized as open-ring structure if one of the C-S bonds is larger than $2.4 \AA$. The results are summarized in Table 3.

Overall $62 \%$ of the trajectories terminate in the singlet manifold from which only $8.8 \%$ reach the $\mathrm{S}_{0}$ state. The triplet states are significantly less populated compared to the dimer. The relative distribution of the $T_{1}$ and the $T_{2}$ state is $8: 5$, while the $T_{3}$ and $S_{2}$ states play no role. This is a first hint for an enhanced life time of the $\mathrm{S}_{1}$ state. For all excited states 
Table 3: Distribution (absolute numbers) of the TP3 B3LYP/DZP trajectories according to the final populated state as well as the final geometry. The shown numbers are statistically not fully converged. The maximum statistical error is $17 \%{ }^{74}$

\begin{tabular}{|c|c|c|c|c|c|c|c|c|c|}
\hline \multirow[b]{2}{*}{ final state } & \multicolumn{8}{|c|}{ final geometry } & \multirow[b]{2}{*}{$\Sigma$} \\
\hline & inner & central & vib. only ${ }^{a}$ & $\mathrm{e}-\mathrm{AA}^{b}$ & e-SS ${ }^{b}$ & $\mathrm{~g}-\mathrm{AA}^{b}$ & $\mathrm{~g}-\mathrm{SA}^{b}$ & $\mathrm{~g}-\mathrm{SS}^{b}$ & \\
\hline $\mathrm{S}_{0}$ & 0 & 0 & 0 & 0 & 1 & 1 & 1 & 0 & 3 \\
\hline $\mathrm{S}_{1}$ & 5 & 4 & 4 & 2 & 0 & 1 & 0 & 0 & 16 \\
\hline $\mathrm{S}_{2}$ & 1 & 1 & 0 & 0 & 0 & 0 & 0 & 0 & 2 \\
\hline $\mathrm{T}_{1}$ & 2 & 1 & 0 & 4 & 0 & 0 & 1 & 1 & 9 \\
\hline $\mathrm{T}_{2}$ & 1 & 3 & 0 & 0 & 0 & 0 & 0 & 0 & 4 \\
\hline$\Sigma$ & 9 & 9 & 4 & 6 & 1 & 2 & 2 & 1 & 34 \\
\hline
\end{tabular}

the open-ring structures are again dominant (53\%). No significant preference towards the cleavage of the inner or the central C-S bonds is observed. The sum of the closed-ring structures in the $\mathrm{T}_{1}$ and $\mathrm{S}_{0}$ states amount to $35 \%$, with an excess of the $e A A$ conformer. In contrast to the TP2 dynamics, nearly $29 \%$ of the analyzed trajectories stay near the FC-region in the $\mathrm{S}_{1}$ state, thereof $12 \%$ show only vibrational dynamics.

The time evolution of the populations of the ground state and the individual excited states are shown in Figure 6 for the TP3.

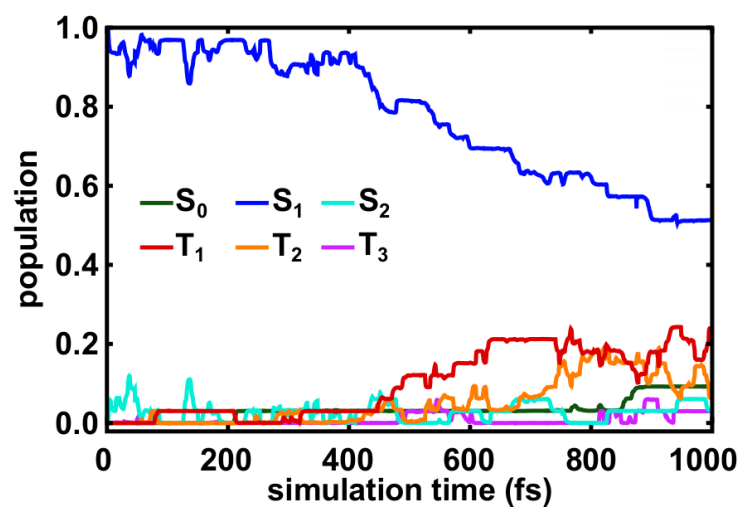

Figure 6: Time evolution of the average populations of the participating excited states for the TP3 trajectories. The ground and the five lowest excited states are displayed.

The relaxation process of the $\mathrm{S}_{1}$ state is significantly slower than in TP2 and again nonexponential. In the first 400 fs the $S_{1}$ population stays nearly constant. The time needed for 
geometrical rearrangement is thus four times longer than for the dimer. After this period, the first two triplet states $\left(\mathrm{T}_{2}\right.$ and $\left.\mathrm{T}_{1}\right)$ can participate in the dynamics and start the nonadiabatic decays. A cascade $\mathrm{S}_{1} \rightarrow \mathrm{T}_{2} \rightarrow \mathrm{T}_{1}$ enables the population transfer back to the ground state $\mathrm{S}_{0}$, comparable to the relaxation dynamics in TP2. Again $\mathrm{S}_{2}$ and $\mathrm{T}_{3}$ do not participate. The ratio between $\mathrm{S}_{0}, \mathrm{~S}_{1}, \mathrm{~T}_{1}$ and $\mathrm{T}_{2}$ after 1 ps simulation time is approx. $15: 50: 25: 10$. The geometrical relaxation process is analyzed with the help of four collective variables associated to ring-opening and the rotation around the inter-ring bonds. The inter-ring rotation is characterized by the two dihedral angles, $\Phi_{S C C S}^{1}$ and $\Phi_{S C C S}^{2}$ (see Fig. 1 b)). The ring-opening is characterized by the averaged CS distance of both inner bonds $\left(r_{C S}^{i n n e r}\right)$ and of both central bonds $\left(r_{C S}^{c e n t r a l}\right)$. The time evolution of these variables is shown in Figure 7.

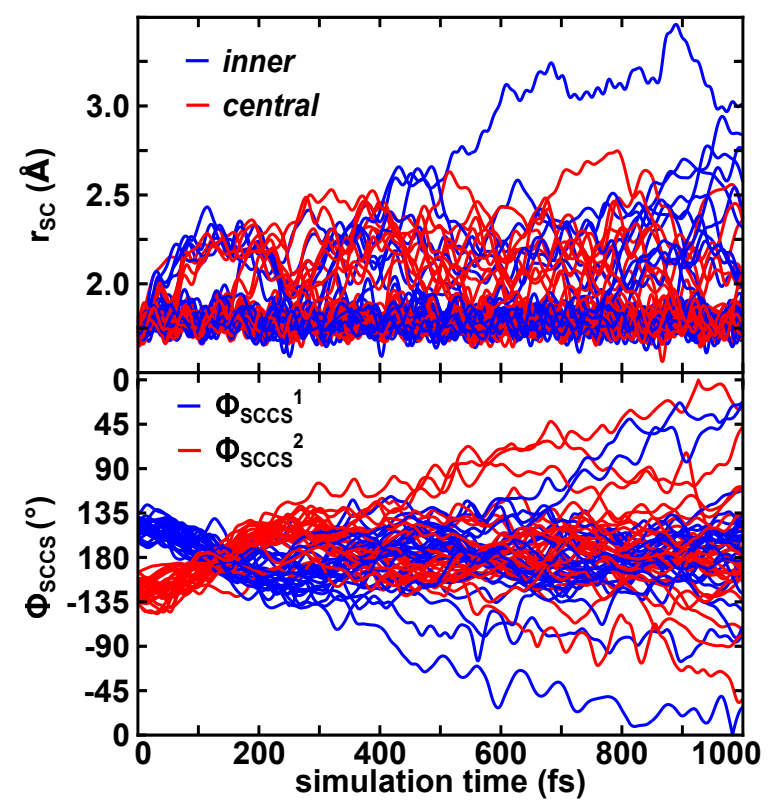

Figure 7: Temporal evolution of the two averaged C-S bonds $\mathrm{r}_{C S}$ and both dihedral angles $\Phi_{S C C S}$ of TP3, up to 1 ps.

The bond coordinates show a similar behavior as well as the dihedral angles, with no clear preferences. In the first 200 fs only a small part of the trajectories undergo ring-opening and therefore the planarization of TP3 may be considered as the dominant geometrical deformation. After this first period more trajectories show open-ring structures, still accompanied by the out-of-plane vibrational motion around the complete planar structure. Overall, the 
bond elongation as well as the inter-ring rotation are less pronounced in the trimer. The inner C-S bonds open slightly further than the central ones, finally leading to longer time scales for inner bonds elongation.

The position of the first hopping events from the $\mathrm{S}_{1}$ state into the triplet manifold were also analyzed. In Figure 8 the position of these hopping events are shown depending on the two averaged C-S bonds $\mathrm{r}_{C S}$ and both dihedral angles $\Phi_{S C C S}$ (see Fig. 1 b)).
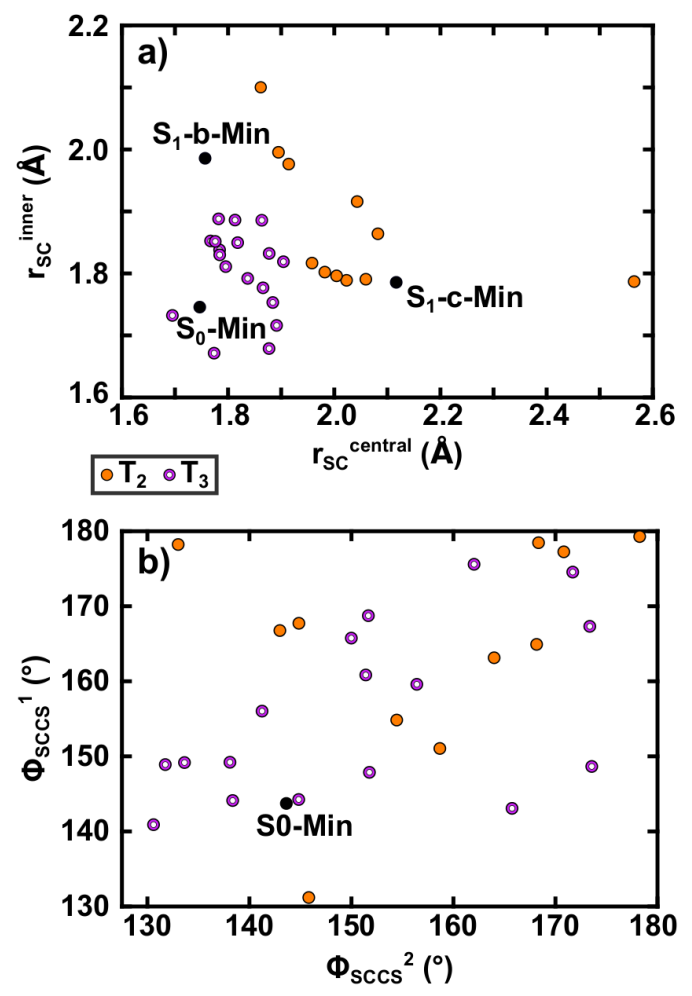

Figure 8: Locations of the TP3 geometries where the first surface hops from the $\mathrm{S}_{1}$ state to triplet states $\left(\mathrm{T}_{2}\right.$ and $\mathrm{T}_{3}$ ) occurred depending on both $\mathrm{r}_{c s}$ distances (a) and both $\Phi_{S C C S}$ dihedral angles (b) for definition see Fig. 1 b). The hopping geometries are displayed in different colors according to the target state of the hopping event. Additionally, relevant B3LYP/DZP optimized geometries are represented in black.

Only intersystem crossing events from the $\mathrm{S}_{1}$ state into the $\mathrm{T}_{2}$ and $\mathrm{T}_{3}$ state are observed. Near the Franck-Condon region, for averaged $\mathrm{r}_{C S}$ values between 1.7-1.9 $\AA$, hops to the $\mathrm{T}_{3}$ state occur. As in the dimer these hops are only intermediate and thus ineffective. Effective hops to the $\mathrm{T}_{2}$ state are again observed for slightly elongated C-S bonds (1.9-2.2 $\AA$ ). More hops into the $\mathrm{T}_{2}$ are observed for central bonds longer than $1.9 \AA$, than for inner bonds. 
For both $\Phi_{S C C S}$ dihedral angles $\left(\right.$ Fig. 8 b)) no clear trend is observed. $T_{2}$-hops occur for dihedral angles in the range $130^{\circ}-180^{\circ}$. Thus also for TP3 bond elongation is the key process. It enables the intersystem crossing into the triplet states as well as the internal conversion back to the ground state. The energy profiles of four illustrative trajectories are shown in Figure 9 .
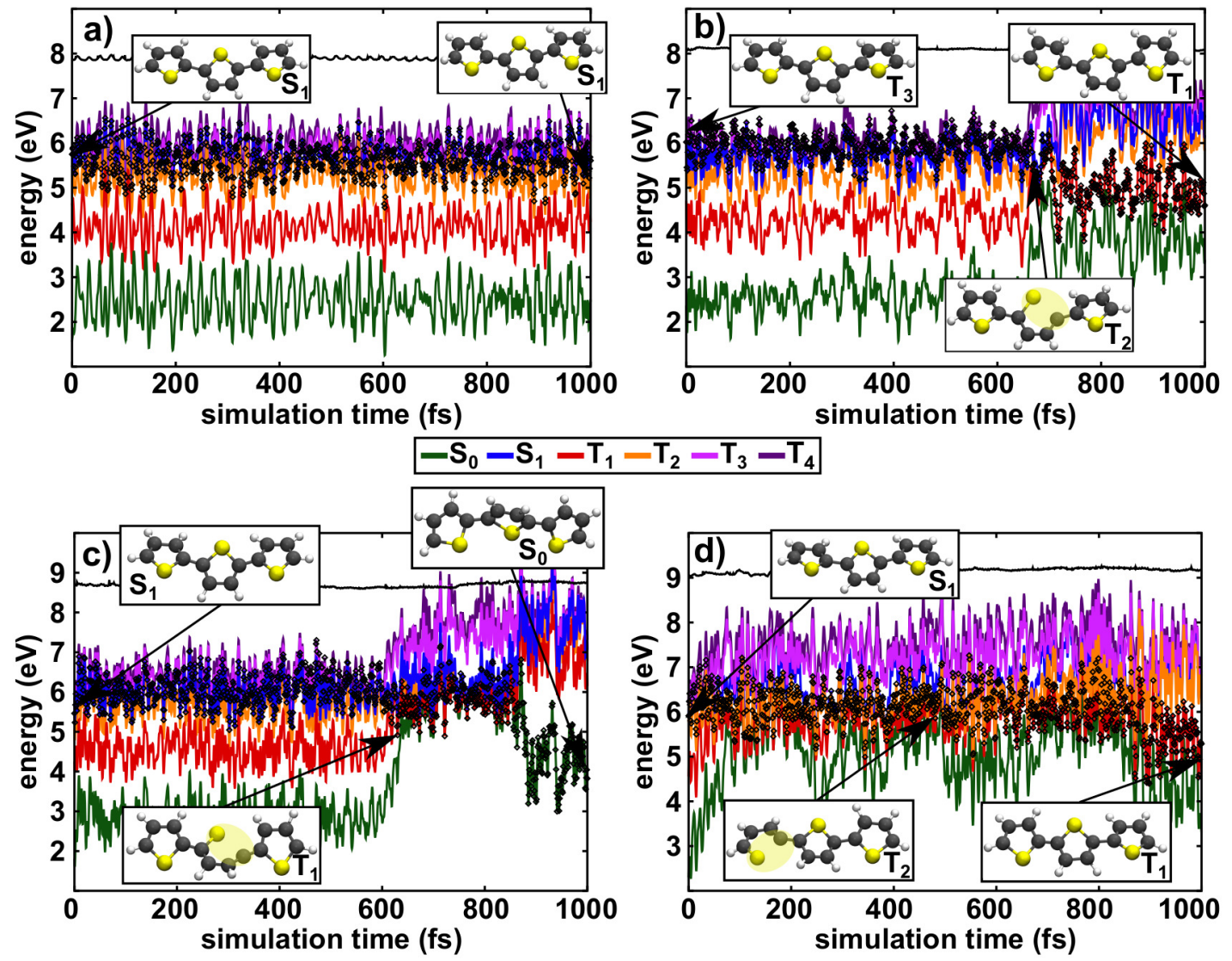

Figure 9: Energy profiles of four representative TP3 trajectories following the ring-opening mechanism. All trajectories were initiated on the $S_{1}$ potential energy surface. The time evolution of the ground and the six lowest excited states are displayed in color, whereas the running state is indicated with black diamonds. The energies are plotted relative to the ground state minimum. Example geometries are given for each trajectory, with the broken carbon sulfur bond highlighted in yellow.

The trajectory of Figure 9 a) only shows vibrational dynamics in the $\mathrm{S}_{1}$ state and presents no non-adiabatic phenomenon. The other trajectories (Figure 9 b), c) and d)) are characterized by intermediate ring-opening events. They can be distinguished either geometrically or regarding their time evolution. In b) and c) one of the central C-S bonds is elongated/broken 
after $600 \mathrm{fs}$. The resulting open-ring structures survive 10 to $100 \mathrm{fs}$ and form closed-ring structures, respectively, in the $\mathrm{T}_{1}$ state (b) or the ground state $\mathrm{S}_{0}(\mathrm{c})$. In the last example d) one of the two inner C-S bonds is broken after approximately $100 \mathrm{fs}$. The resulting open-ring structure persists for about 700 fs allowing hops between the $S_{1}, T_{1}$ and $T_{2}$ state. This is in good agreement with the observed slight preference for inner bond elongation (compare with Fig. 7 top). The excited state lifetime is globally longer compared to dimer and monomer. This is due to (i) a decrease in spin-orbit couplings as the average C-S bond-elongation is smaller and (ii) an increase in the barrier towards bond elongation. Like the dimer, closedring structures are formed in the $\mathrm{T}_{1}$ state opening the path to phosphorescence.

\section{Conclusions}

The relaxation mechanisms of gas-phase irradiated oligothiophene have been unraveled and critically characterized by means of non-adiabatic surface-hopping dynamics. The TDDFT level of theory has been employed to calculate the energies, gradients as well as non-adiabatic and spin-orbit couplings on-the-fly along the trajectories. This level of theory has been carefully benchmarked against high level theoretical as well as experimental data, assuring a qualitatively correct and almost quantitative description of all states involved. In our interpretation we took into account possible limitations of the method in describing the IC back to the ground state. In partial contrast with previous studies performed without the explicit inclusion of the spin-orbit coupling elements, the simulation presents a quite complex scenario in which different competitive pathways are active. Moreover, the influence of the triplet state manifold appears crucial in driving the process. Indeed, in the case of TP2 more than $60 \%$ of the trajectories ends in the triplet manifold after 1 ps.

The strong influence of the triplet states was also observed for the case of the thiophene monomer ${ }^{24}$ where it was connected to the carbon sulfur bond cleavage, leading to ringopening and to a region of singlet triplet quasi-degeneracy and increased spin-orbit couplings. As schematically reported in Figure 10, a similar picture holds also in the case of 
oligothiophene, even though the complexity is strongly increased.
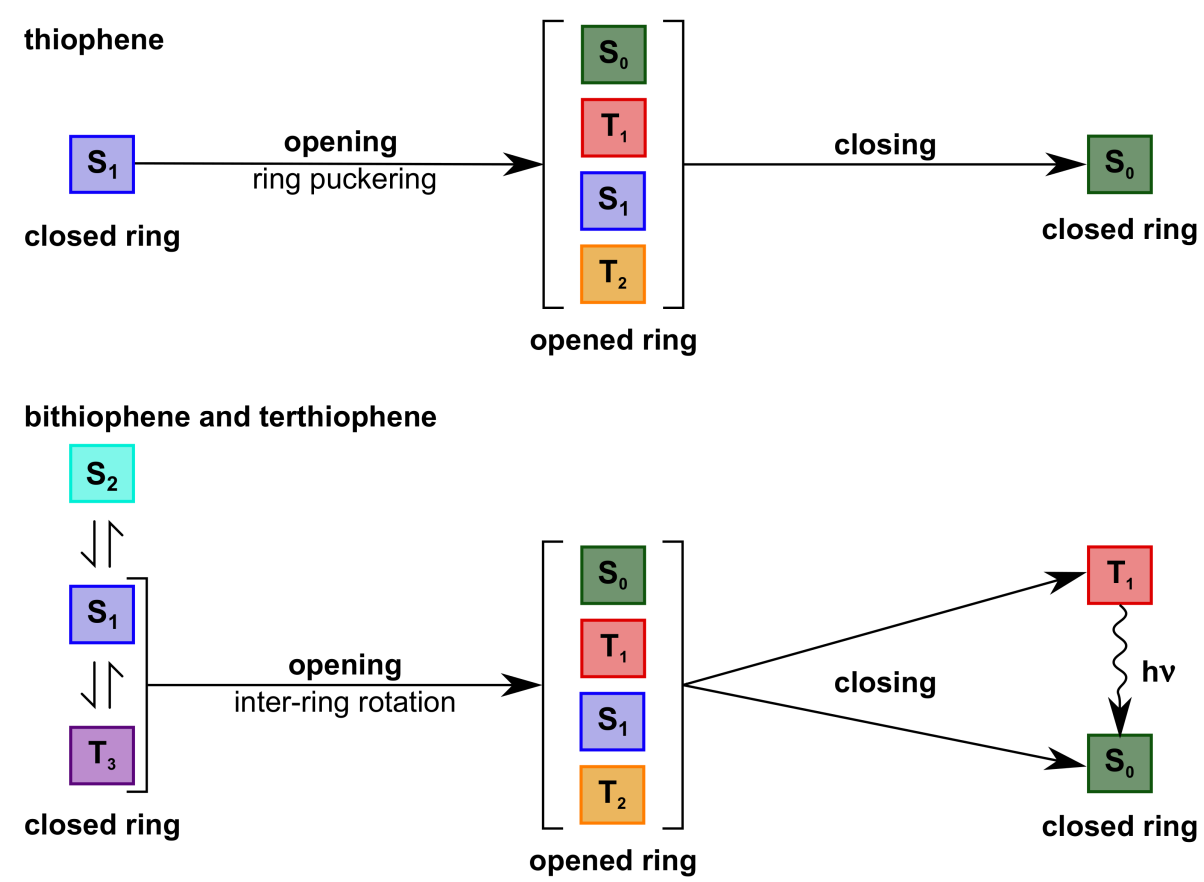

Figure 10: Schematic representation of the pathways active in the excited state relaxation of thiophene monomer compared with bithiophene (TP2) and terthiophene (TP3), as rationalized from non-adiabatic dynamics.

After excitation to the $\mathrm{S}_{1}$ state, the relaxation process for the majority of the trajectories is described by ring-opening and inter-ring rotation, leading to an extended region of quasidegeneracy between the triplet and singlet potential energy surfaces. Hence, in this region a complex equilibrium between the different states involved is established, characterized by frequent back and forwards non-adiabatic events. Eventually the system can further evolve to the ring closure that can take place either on the $S_{0}$ or the $T_{1}$ state. In addition, the ring-opening and subsequent closure is accompanied by a high flexibility of the intermediate molecular systems and indeed trans-cis isomerization is observed. The conclusions for the TP3 are qualitatively similar, even if in this case the ring-opening is less pronounced and one should speak of a carbon-sulfur bond elongation rather than of bond-cleavage. With increasing chainlenght the excitation is delocalized over a larger number of bonds, so each individual bond is weakened less. 
Our results clearly identify the bond cleavage (or elongation) as the key coordinate driving the relaxation, also via the population of the triplet manifold. This process involves rather large rearrangements of the molecular geometries all along the 1 ps trajectory, even leading to a certain amount of trans-cis isomerization. The formation of closed-ring structures in the $\mathrm{T}_{1}$ state of the dimer and trimer is a prominent difference to the thiophene monomer and we believe it is the reason why phosphorescence is experimentally observable for longer thiophene chains. The efficiency of the ISC is reduced for longer thiophene chains as the driving coordinate, the carbon sulfur bond cleavage, is hindered. Hence, we have provided a consistent description of the photophysics of oligomeric thiophene, taking into account the coupling between intersystem crossing and the geometrical reorganization. However, it has to be underlined that our simulations have been performed in gas phase. The influence of a viscose environment, such as polymeric matrices, can be quite important since the geometric reorganization may be hampered. In the near future we plan to tackle this problem by performing $\mathrm{QM} / \mathrm{MM}$ non-adiabatic dynamics in thiophene oligomers embedded in rigid polymeric matrices.

\section{Acknowledgement}

Financial support of this work from Deutsche Forschungsgemeinschaft through the SFB749 and the excellence cluster 'Munich-Centre for Advanced Photonics' (MAP) is gratefully acknowledged. The computational results presented have been partially achieved using the Vienna Scientific Cluster (VSC). M.M. is grateful to the Universidad de La Rioja (UR) for a postdoctoral grant. A.M. also acknowledge French CNRS and Université de Lorraine for support. S.M. and L.G. acknowledge funding from the Austrian Science Fund (FWF) within project I2883 (DeNeTheor) and the University of Vienna.

\section{Supporting Information Available}

Validation of the static quantum chemical calculations and comparison with the reference 
values. Time series of some relevant geometrical coordinates up to the ps time scale and distribution of the hops between the states. Coordinates of the optimized geometries of the relevant critical points. Evolution of the average population of the states and of the hops between states obtained from the full set of trajectories. This material is available free of charge via the Internet at http://pubs . acs.org/.

\section{References}

(1) Skotheim, T. A., Reynolds, J. R., Eds. Handbook of Conducting Polymers, 3rd ed.; CRC Press: Boca Raton, FL, 2007.

(2) Perepichka, I. F., Perepichka, D. F., Eds. Handbook of Thiophene-Based Materials: Applications in Organic Electronics and Photonics; John Wiley \& Sons Ltd: Chichester, UK, 2007.

(3) Yu, W.; Donohoo-Vallett, P. J.; Zhou, J.; Bragg, A. E. Ultrafast photo-induced nuclear relaxation of a conformationally disordered conjugated polymer probed with transient absorption and femtosecond stimulated Raman spectroscopies. J. Chem. Phys. 2014, 141, 044201.

(4) Wang, C.; Angelella, M.; Doyle, S. J.; Lytwak, L. A.; Rossky, P. J.; Holliday, B. J.; Tauber, M. J. Resonance Raman Spectroscopy of the $T_{1}$ Triplet Excited State of Oligothiophenes. J. Phys. Chem. Lett. 2015, 6, 3521-3527.

(5) Thomas, A. K.; Brown, H. A.; Datko, B. D.; Garcia-Galvez, J. A.; Grey, J. K. Interchain Charge-Transfer States Mediate Triplet Formation in Purified Conjugated Polymer Aggregates. J. Phys. Chem. C 2016, 120, 23230-23238.

(6) Becker, R. S.; Seixas de Melo, J.; Maçanita, A. L.; Elisei, F. Comprehensive Evaluation of the Absorption, Photophysical, Energy Transfer, Structural, and Theoretical 
Properties of $\alpha$-Oligothiophenes with One to Seven Rings. J. Phys. Chem. 1996, 100, $18683-18695$.

(7) Turro, N. J., Ramamurthy, V., Scaiano, J., Eds. Modern Molecular Photochemistry, 1st ed.; University Science Books: Mill Valley, CA, 2010.

(8) Rupert, B. L.; Mitchell, W. J.; Ferguson, A. J.; Köse, M. E.; Rance, W. L.; Rumbles, G.; Ginley, D. S.; Shaheen, S. E.; Kopidakis, N. Low-bandgap thiophene dendrimers for improved light harvesting. J. Mater. Chem. 2009, 19, 5311-5324.

(9) Zhang, F.; Wu, D.; Xu, Y.; Feng, X. Thiophene-based conjugated oligomers for organic solar cells. J. Mater. Chem. 2011, 21, 17590-17600.

(10) Beaujuge, P. M.; Fréchet, J. M. J. Molecular Design and Ordering Effects in $\pi$ Functional Materials for Transistor and Solar Cell Applications. J. Am. Chem. Soc. 2011, 133, 20009-20029.

(11) Chi, D.; Qu, S.; Wang, Z.; Wang, J. High efficiency P3HT:PCBM solar cells with an inserted PCBM layer. J. Mater. Chem. C 2014, 2, 4383.

(12) Hu, H.; Jiang, K.; Yang, G.; Liu, J.; Li, Z.; Lin, H.; Liu, Y.; Zhao, J.; Zhang, J.; Huang, F.; Qu, Y.; Ma, W.; Yan, H. Terthiophene-Based D-A Polymer with an Asymmetric Arrangement of Alkyl Chains That Enables Efficient Polymer Solar Cells. J. Am. Chem. Soc. 2015, 137, 14149-14157.

(13) Gigli, G.; Inganäs, O.; Anni, M.; De Vittorio, M.; Cingolani, R.; Barbarella, G.; Favaretto, L. Multicolor oligothiophene-based light-emitting diodes. Appl. Phys. Lett. 2001, 78, 1493-1495.

(14) Mazzeo, M.; Pisignano, D.; Favaretto, L.; Barbarella, G.; Cingolani, R.; Gigli, G. Bright oligothiophene-based light emitting diodes. Synth. Met. 2003, 139, 671-673. 
(15) Irie, M.; Fukaminato, T.; Matsuda, K.; Kobatake, S. Photochromism of Diarylethene Molecules and Crystals: Memories, Switches, and Actuators. Chem. Rev. 2014, 114, $12174-12277$.

(16) Barbarella, G.; Zambianchi, M.; Ventola, A.; Fabiano, E.; Della Sala, F.; Gigli, G.; Anni, M.; Bolognesi, A.; Polito, L.; Naldi, M.; Capobianco, M. Bright Oligothiophene N -Succinimidyl Esters for Efficient Fluorescent Labeling of Proteins and Oligonucleotides. Bioconjugate Chem. 2006, 17, 58-67.

(17) Ho, H.-A.; Najari, A.; Leclerc, M. Optical Detection of DNA and Proteins with Cationic Polythiophenes. Acc. Chem. Res. 2008, 41, 168-178.

(18) Zambianchi, M.; Maria, F. D.; Cazzato, A.; Gigli, G.; Piacenza, M.; Sala, F. D.; Barbarella, G. Microwave-Assisted Synthesis of Thiophene Fluorophores, Labeling and Multilabeling of Monoclonal Antibodies, and Long Lasting Staining of Fixed Cells. J. Am. Chem. Soc. 2009, 131, 10892-10900.

(19) Bhaskar, A.; Ramakrishna, G.; Hagedorn, K.; Varnavski, O.; Mena-Osteritz, E.; Bäuerle, P.; Goodson, T. Enhancement of Two-Photon Absorption Cross-Section in Macrocyclic Thiophenes with Cavities in the Nanometer Regime. J. Phys. Chem. B 2007, 111, 946-954.

(20) Zhou, H.; Zhou, F.; Tang, S.; Wu, P.; Chen, Y.; Tu, Y.; Wu, J.; Tian, Y. Two-photon absorption dyes with thiophene as $\pi$-electron bridge: Synthesis, photophysical properties and optical data storage. Dyes Pigment. 2012, 92, 633-641.

(21) Turan, H. T.; Eken, Y.; Marazzi, M.; Pastore, M.; Aviyente, V.; Monari, A. Assessing One- and Two-Photon Optical Properties of Boron Containing Arenes. J. Phys. Chem. C 2016, 120, 17916-17926.

(22) Huix-Rotllant, M.; Tamura, H.; Burghardt, I. Concurrent Effects of Delocalization and 
Internal Conversion Tune Charge Separation at Regioregular Polythiophene-Fullerene Heterojunctions. J. Phys. Chem. Lett. 2015, 6, 1702-1708.

(23) Kölle, P.; Schnappinger, T.; de Vivie-Riedle, R. Deactivation pathways of thiophene and oligothiophenes: internal conversion versus intersystem crossing. Phys. Chem. Chem. Phys. 2016, 18, 7903-7915.

(24) Schnappinger, T.; Kölle, P.; Marazzi, M.; Monari, A.; González, L.; de Vivie-Riedle, R. Ab initio molecular dynamics of thiophene: the interplay of internal conversion and intersystem crossing. Phys. Chem. Chem. Phys. 2017, 19, 25662-25670.

(25) Weinkauf, R.; Lehr, L.; Schlag, E. W.; Salzmann, S.; Marian, C. M. Ultrafast dynamics in thiophene investigated by femtosecond pump probe photoelectron spectroscopy and theory. Phys. Chem. Chem. Phys. 2008, 10, 393-404.

(26) Lap, D. V.; Grebner, D.; Rentsch, S. Femtosecond Time-Resolved Spectroscopic Studies on Thiophene Oligomers. J. Phys. Chem. A 1997, 101, 107-112.

(27) Paa, W.; Yang, J.-P.; Rentsch, S. Intersystem crossing in oligothiophenes studied by fs time-resolved spectroscopy. Appl. Phys. B 2000, 71, 443-449.

(28) Barclay, M. S.; Quincy, T. J.; Williams-Young, D. B.; Caricato, M.; Elles, C. G. Accurate Assignments of Excited-State Resonance Raman Spectra: A Benchmark Study Combining Experiment and Theory. J. Phys. Chem. A 2017, 121, 7937-7946.

(29) Rubio, M.; Merchán, M.; Ortí, E.; Roos, B. O. A theoretical study of the electronic spectrum of bithiophene. J. Chem. Phys. 1995, 102, 3580.

(30) Rubio, M.; Merchán, M.; Pou-Amérigo, R.; Ortí, E. The Low-Lying Excited States of 2,2'-Bithiophene: A Theoretical Analysis. ChemPhysChem 2003, 4, 1308-1315.

(31) Rubio, M.; Merchán, M.; Ortí, E. A Theoretical Study on the Low-Lying Excited States 
of 2,2':5',2"-Terthiophene and 2,2':5',2":5",2"'-Quaterthiophene. ChemPhysChem 2005, 6, 1357-1368.

(32) Siegert, S.; Vogeler, F.; Marian, C. M.; Weinkauf, R. Throwing light on dark states of $\alpha$-oligothiophenes of chain lengths 2 to 6 : radical anion photoelectron spectroscopy and excited-state theory. Phys. Chem. Chem. Phys. 2011, 13, 10350-10363.

(33) Fazzi, D.; Barbatti, M.; Thiel, W. Modeling ultrafast exciton deactivation in oligothiophenes via nonadiabatic dynamics. Phys. Chem. Chem. Phys. 2015, 17, 7787-7799.

(34) Prlj, A.; Curchod, B. F. E.; Corminboeuf, C. Excited state dynamics of thiophene and bithiophene: new insights into theoretically challenging systems. Phys. Chem. Chem. Phys. 2015, 17, 14719-14730.

(35) Lin, J. B.; Jin, Y.; Lopez, S. A.; Druckerman, N.; Wheeler, S. E.; Houk, K. N. Torsional Barriers to Rotation and Planarization in Heterocyclic Oligomers of Value in Organic Electronics. J. Chem. Theory Comput. 2017, 13, 5624-5638.

(36) Richter, M.; Marquetand, P.; González-Vázquez, J.; Sola, I.; González, L. SHARC: ab initio Molecular Dynamics with Surface Hopping in the Adiabatic Representation Including Arbitrary Couplings. J. Chem. Theory Comput. 2011, 7, 1253-1258.

(37) Mai, S.; Marquetand, P.; González, L. A General Method to Describe Intersystem Crossing Dynamics in Trajectory Surface Hopping. Int. J. Quantum Chem. 2015, 115, $1215-1231$.

(38) Mai, S.; Marquetand, P.; González, L. Nonadiabatic dynamics: The SHARC approach. Wiley Interdiscip. Rev.: Comput. Mol. Sci. 2018, 0, e1370.

(39) Mai, S.; Richter, M.; Heindl, M.; Menger, M. F. S. J.; Atkins, A.; Ruckenbauer, M.; Plasser, F.; Oppel, M.; Marquetand, P.; González, L. SHARC2.0: Surface Hopping 
Including Arbitrary Couplings - Program Package for Non-Adiabatic Dynamics. sharcmd.org, 2018.

(40) Richter, M.; Mai, S.; Marquetand, P.; González, L. Ultrafast intersystem crossing dynamics in uracil unravelled by ab initio molecular dynamics. Phys. Chem. Chem. Phys. 2014, 16, 24423-24436.

(41) Cao, J.; Xie, Z.-Z.; Yu, X. Excited-state dynamics of oxazole: A combined electronic structure calculations and dynamic simulations study. Chem. Phys. 2016, 474, $25-35$.

(42) Marazzi, M.; Mai, S.; Roca-Sanjuán, D.; Delcey, M. G.; Lindh, R.; González, L.; Monari, A. Benzophenone Ultrafast Triplet Population: Revisiting the Kinetic Model by Surface-Hopping Dynamics. J. Phys. Chem. Lett. 2016, 7, 622-626.

(43) Mai, S.; Pollum, M.; Martínez-Fernández, L.; Dunn, N.; Marquetand, P.; Corral, I.; Crespo-Hernández, C. E.; González, L. The origin of efficient triplet state population in sulfur-substituted nucleobases. Nat. Commun. 2016, \%, 13077.

(44) Mai, S.; Marquetand, P.; González, L. Intersystem Crossing Pathways in the Noncanonical Nucleobase 2-Thiouracil: A Time-Dependent Picture. J. Phys. Chem. Lett. 2016, ๆ, 1978-1983.

(45) Hirata, S.; Head-Gordon, M. Time-dependent density functional theory within the Tamm-Dancoff approximation. Chem. Phys. Lett. 1999, 314, 291-299.

(46) Baerends, E. J. et al. ADF2017, SCM, Theoretical Chemistry, Vrije Universiteit, Amsterdam, The Netherlands, https://www.scm.com.

(47) te Velde, G.; Bickelhaupt, F. M.; Baerends, E. J.; Fonseca Guerra, C.; van Gisbergen, S. J. A.; Snijders, J. G.; Ziegler, T. Chemistry with ADF. J. Comput. Chem. 2001, 22, $931-967$. 
(48) Lee, C.; Yang, W.; Parr, R. G. Development of the Colle-Salvetti correlation-energy formula into a functional of the electron density. Phys. Rev. B 1988, 37, 785-789.

(49) Becke, A. D. Density-functional thermochemistry. III. The role of exact exchange. J. Chem. Phys. 1993, 98, 5648-5652.

(50) Stephens, P. J.; Devlin, F. J.; Chabalowski, C. F.; Frisch, M. J. Ab Initio Calculation of Vibrational Absorption and Circular Dichroism Spectra Using Density Functional Force Fields. J. Chem. Phys. 1994, 98, 11623-11627.

(51) Grimme, S.; Antony, J.; Ehrlich, S.; Krieg, H. A consistent and accurate ab initio parametrization of density functional dispersion correction (DFT-D) for the 94 elements H-Pu. J. Chem. Phys. 2010, 132, 154104.

(52) Neese, F. The ORCA program system. Wiley Interdiscip. Rev.: Comput. Mol. Sci. 2012, 2, 73-78.

(53) Van Lenthe, E.; Baerends, E. J. Optimized Slater-type basis sets for the elements 1-118. J. Comput. Chem. 2003, 24, 1142-1156.

(54) Becke, A. D. A multicenter numerical integration scheme for polyatomic molecules. J. Chem. Phys. 1988, 88, 2547-2553.

(55) Franchini, M.; Philipsen, P. H. T.; Visscher, L. The Becke Fuzzy Cells Integration Scheme in the Amsterdam Density Functional Program Suite. J. Comput. Chem. 2013, 34, 1819-1827.

(56) Franchini, M.; Philipsen, P. H. T.; van Lenthe, E.; Visscher, L. Accurate Coulomb Potentials for Periodic and Molecular Systems through Density Fitting. J. Chem. Theory Comput. 2014, 10, 1994-2004.

(57) van Lenthe, E.; Baerends, E. J.; Snijders, J. G. Relativistic regular two-component Hamiltonians. J. Chem. Phys. 1993, 99, 4597-4610. 
(58) van Lenthe, E.; Baerends, E. J.; Snijders, J. G. Relativistic total energy using regular approximations. J. Chem. Phys. 1994, 101, 9783-9792.

(59) van Lenthe, E.; Snijders, J. G.; Baerends, E. J. The zero-order regular approximation for relativistic effects: The effect of spin-orbit coupling in closed shell molecules. $J$. Chem. Phys. 1996, 105, 6505-6516.

(60) van Lenthe, E.; van Leeuwen, R.; Baerends, E. J.; Snijders, J. G. Relativistic regular two-component Hamiltonians. Int. J. Quantum Chem. 1996, 57, 281-293.

(61) van Lenthe, E.; Ehlers, A.; Baerends, E.-J. Geometry optimizations in the zero order regular approximation for relativistic effects. J. Chem. Phys. 1999, 110, 8943-8953.

(62) Wang, F.; Ziegler, T. A simplified relativistic time-dependent density-functional theory formalism for the calculations of excitation energies including spin-orbit coupling effect. J. Chem. Phys. 2005, 123, 154102.

(63) Duarte, H. A.; Dos Santos, H. F.; Rocha, W. R.; De Almeida, W. B. Improved quantum mechanical study of the potential energy surface for the bithiophene molecule. J. Chem. Phys. 2000, 113, 4206-4215.

(64) Ciofalo, M.; Manna, G. L. Ab initio conformational study of $2,2^{\prime}: 5^{\prime}, 2$ "-terthiophene. Chem. Phys. Lett. 1996, 263, 73-78.

(65) Millefiori, S.; Alparone, A.; Millefiori, A. Conformational properties of thiophene oligomers. J. Heterocyclic Chem. 2000, 37, 847-853.

(66) Liu, F.; Zuo, P.; Meng, L.; Zheng, S. J. On the optical properties of thiophene oligomers: configuration interaction study on their ground $\left(\mathrm{S}_{0}\right)$ and first singlet excited $\left(\mathrm{S}_{1}\right)$ states. THEOCHEM 2005, 726, 161-169.

(67) Mai, S.; Marquetand, P.; Richter, M.; González-Vázquez, J.; González, L. Singlet and 
Triplet Excited-State Dynamics Study of the Keto and Enol Tautomers of Cytosine. ChemPhysChem 2013, 14, 2920-2931.

(68) Granucci, G.; Persico, M.; Toniolo, A. Direct semiclassical simulation of photochemical processes with semiempirical wave functions. J. Chem. Phys. 2001, 114, 10608-10615.

(69) Plasser, F.; Ruckenbauer, M.; Mai, S.; Oppel, M.; Marquetand, P.; González, L. Efficient and Flexible Computation of Many-Electron Wave Function Overlaps. J. Chem. Theory Comput. 2016, 12, 1207-1219.

(70) Granucci, G.; Persico, M. Critical appraisal of the fewest switches algorithm for surface hopping. J. Chem. Phys. 2007, 126, 134114.

(71) Belletête, M.; Leclerc, M.; Durocher, G. Potentialities of Semiempirical Calculations (AMPAC and INDO/S) in Determining the Conformation and Electronic Properties of 2,2'-Bithiophene: A New Joint Experimental and Theoretical Approach. J. Phys. Chem. 1994, 98, 9450-9456.

(72) Grebner, D.; Helbig, M.; Rentsch, S. Size-Dependent Properties of Oligothiophenes by Picosecond Time-Resolved Spectroscopy. J. Phys. Chem. 1995, 99, 16991-16998.

(73) DiCésare, N.; Belletête, M.; Marrano, C.; Leclerc, M.; Durocher, G. Intermolecular Interactions in Conjugated Oligothiophenes. 1. Optical Spectra of Terthiophene and Substituted Terthiophenes Recorded in Various Environments. J. Phys. Chem. A 1999, 103, 795-802.

(74) Crespo-Otero, R.; Barbatti, M. Recent Advances and Perspectives on Nonadiabatic Mixed Quantum-Classical Dynamics. Chemical Reviews 0, 0, null, PMID: 29767966.

(75) Rentsch, S.; Yang, J. P.; Paa, W.; Birckner, E.; Schiedt, J.; Weinkauf, R. Size dependence of triplet and singlet states of $\alpha$-oligothiophenes. Phys. Chem. Chem. Phys. 1999, 1, 1707-1714. 
Graphical TOC Entry

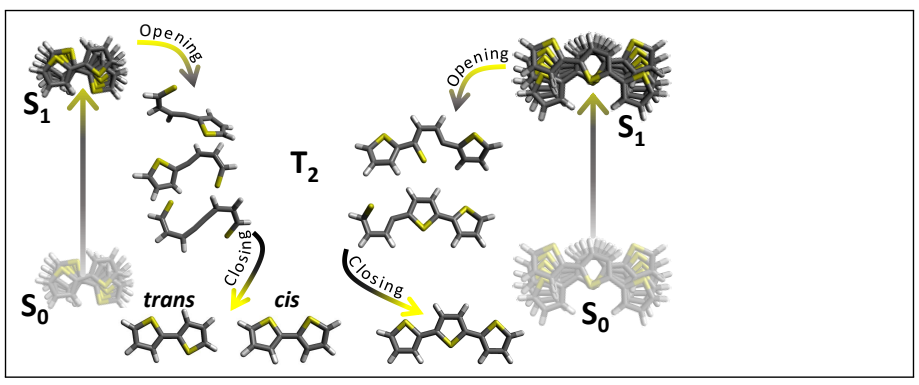

